\title{
Fetal Arrhythmias: A Clinical Review
}

\author{
Julia Hajdu, Barbara Pete, Agnes Harmath, Valeria Váradi, Zoltán Papp
}

First Department of Obstetrics and Gynecology, Semmelweis University Medical Faculty, Budapest, Hungary

Correspondence: Julia Hajdu, First Department of Obstetrics and Gynecology, Semmelweis University Medical Faculty, Baross street 27, 1088 Budapest, Hungary, e-mail: hajdu@noi1.sote.hu

\begin{abstract}
Fetal rhythm abnormalities occur in $2 \%$ of pregnancies. They are usually identified by the obstetrician or midwifes after 20 weeks. There are four different methods used to assess fetal arrhythmias: scalp electrodes attached to electrocardiographic recordings, magnetocardiography (FMCG), fetal electrocardiographic recordings from the maternal abdomen, and fetal echocardiography (M-mode, pulsed-Doppler, Tissue-Doppler). In everyday practice the Doppler method was found to be the most useful method in the diagnosis and therapy of fetal arrhythmias. Doppler derived mechanical PR interval raised the possibility of refining the prenatal diagnosis of $A V$ conduction abnormalities. A PR interval of $>150 \mathrm{~ms}$ on Doppler, FMCG or postnatal ECG has been determined to be prolonged. Extrasystoles are most common cause of fetal arrhythmias, and are most often premature atrial contractions (PACs), what are usually identified in third trimester fetuses and their frequency may be highly variable. These are usually benign, resolving just before or shortly after birth. The follow-up is necessary, because some (1-3\%) of affected fetuses have intermittent runs of supraventricular tachycardia. Ventricular tachycardia is rare during fetal life. With echocardiography in the setting of fetal tachycardia the findings of atrioventricular dissociation with a ventricular rate that is faster than the atrial rate suggests ventricular tachycardia. If there is $1: 1$ retrograde conduction it is impossible to distinguish between ventricular and supraventricular tachycardia. Atrial flutter accounted for $26.2 \%$ of all cases of fetal tachyarrhythmias and supraventricular tachycardia for $73.2 \%$. Fouron and coworkers proposed to plan the management of the fetal tachyarrhythmia based on analysis of pulsed-Doppler recordings of fetal heart's blood flow. They determined short V-A tachycardia, when V-A (ventriculoatrial period) was shorter than A$V$ (atrio-ventricular period) period. In the therapy of fetal supraventricular tachycardia there are different protocols, the most commonly used drugs are: digoxin, sotalol, amiodarone, flecainide. Persistent fetal sinus bradycardia is a rare condition and has been reported with central nervous system abnormalities, maternal treatment with beta blockers, excessive vagal tone, hydrops, long QT syndrome, intrauterine growth retardation and could be a sign of maternal anti-SSA/Ro antibodies. Prenatal sinus bradycardia or recognition of 2 nd degree AV block may lead to early detection and treatment of long QT syndrome. Early detection of incomplete AV block, in cases of maternal anti SSA, SSB autoantibodies, successfully identifies a group at highest risk developing permanent AV block. The anti-inflammatory effects of dexamethasone might have interrupted on-going damage of the conduction system secondary to maternal autoantibodies. If the fetal arrhythmia resulted fetal hydrops, the mortality is high and the risk of late neurological morbidity must be taken into consideration. As a result of close follow-up, transplacentar treatment and well-organized perinatal management,
\end{abstract}

the survival of sustained fetal arrhythmia significantly improved $(50 \%$ versus $15 \%$ ).

Keywords: Fetal arrhythmias, Doppler, follow-up.

\section{DEVELOPMENT OF THE CONDUCTION SYSTEM}

In avian embryos, a pacemaker is first established in the inflow tract at the straight heart tube stage. From the onset of the function a cranial-to caudal axis is established both for pacemaker dominance and for intrinsic beat. Transplantation experiments in animal model have shown that the polarity along this axis of contraction is not fixed and can adapt to a new position. When the embryonic heart first begins to contract, it has no specialized conduction system. It is thought that the cardiac conduction system are derived from independent parent cells linked together to establish the integrated conduction system of the heart. Terminal differentiation of conduction tissue occurs late in development. ${ }^{1}$

It was found, that there is an important role for two transitional zones the sinus venosus and the primary ring in the development of the conduction system. The incorporation of the sinus venosus into the atrium showed the embryonic existence of three internodal pathways between the sinoatrial and the atrioventricular node and the relation of the pulmonary venous system in the dorsal left atrial wall with the primitive conduction system. Studies on CCS lacZ mouse showed the development of the interatrial Bachmann's bundle and the continuation of the right ventricular moderator band with the right atrioventricular ring bundle. It was postulated a role for neural crest cells in the induction of the central conduction system and for the epicardium-derived cells in differentiation of the Purkinje network. ${ }^{2}$

By 6 weeks postconception AV synchrony can be demonstrated using standard Doppler techniques. The heart rate is 150 bpm by 14 weeks, by 20 weeks it is $140 \mathrm{bpm}$ with a gradual decrease to $130 \mathrm{bpm}$ by term. Fetal rhythm abnormalities occur in $2 \%$ of pregnancies. They are usually identified by the obstetrician or midwifes. Most fetal arrhythmias are detected after 20 weeks. ${ }^{3}$ 


\section{METHODS OF THE INVESTIGATION}

There are four different methods used to assess fetal arrhythmias: scalp electrodes attached to electrocardiographic recordings, magnetocardiography (FMCG), fetal electrocardiographic recordings from the maternal abdomen, and fetal echocardiography (M-mode, pulsed-Doppler, tissue-Doppler).

The usefulness of the invasive method, scalp electrodes attached to electrocardiographic recordings is limited, because it is possible to use only at ruptured membranes. A number of studies have demonstrated the efficacy of fetal magnetocardiography for the evaluation of normal and abnormal fetal rhythms. ${ }^{4}$

Vector magnetocardiography was potentially useful for standardization of the FMCG waveforms and to provide a more complete and accurate analysis of fetal arrhythmias. ${ }^{5}$ FMCG provides a noninvasive means of analyzing complex tachyarrhythmia in utero, with efficacy approaching that of postnatal electrocardiographic rhythm monitoring. ${ }^{6}$ The disadvantage of fetal magnetocardiography, that it is expensive.

The fetal electrocardiography is not performed routinely because of the limited quality of the surface fetal electrocardiographic signal. With newer equipment it was found, that electrical PR interval was better than mechanical PR interval at differentiating between normal and prolonged PR in the fetus and the reproducibility of the PR interval using noninvasive FECG was significantly better than that of the Doppler methods. ${ }^{7-9}$

In the fetal heart electrical activity can be inferred from the timing of the mechanical events. With M-mode method the simultaneous recording of the movement of the right atrial wall or the opening and closing of the atrioventricular valves and of the ventricular wall or the opening and closing of the aortic valves makes it possible to determine the relationship of the atrial and ventricular electric activity. The fetal M mode echocardiogram has its limitations: The measurement of the fetal atrioventricular conduction time is not accurate, the loss of clear markers of atrial and ventricular contractions in hydropic fetuses with hypocontractile myocardium and the poor picture resolution related to factors such as maternal obesity, fetal position, or polyhydramnios.

The Doppler method was found to be superior compared with the M-mode approach when to measure fetal atrioventricular time intervals. There are three different places where it is possible to measure mechanical PR interval. The MV-Ao Doppler method is recorded in 5-chamber view with the septum in a vertical position velocities generated by both in inflow of blood through the mitral valve (MV) during diastole and by the outflow through the left ventricular outlet (Ao) during systole. The PR interval is measured from the intersection of the mitral $\mathrm{E}$ and $\mathrm{A}$ waves to the beginning of the ventricular ejection wave (Fig. 1). Another Doppler techniques simultaneous recording of superior vena cava (SVC) and ascending aorta (AA) flow velocity waveforms (Fig. 2). Pulsed Doppler sample volume is adjusted to encompass both vessels. On these simultaneous recordings AV time interval is measured from the beginning of the retrograde venous $A$ wave to the beginning of the aortic ejection wave. ${ }^{10}$ In the normal fetus, when the SVC/AA Doppler approach was used, gestational age was the only independent variable that influences the AV time intervals. The difference between the AV time intervals at 17-20 weeks and 36-39 weeks is mild, with no significant difference. Maximum AV conduction time was found to be 135 ms in the normal population. ${ }^{9-12}$ With another method sample

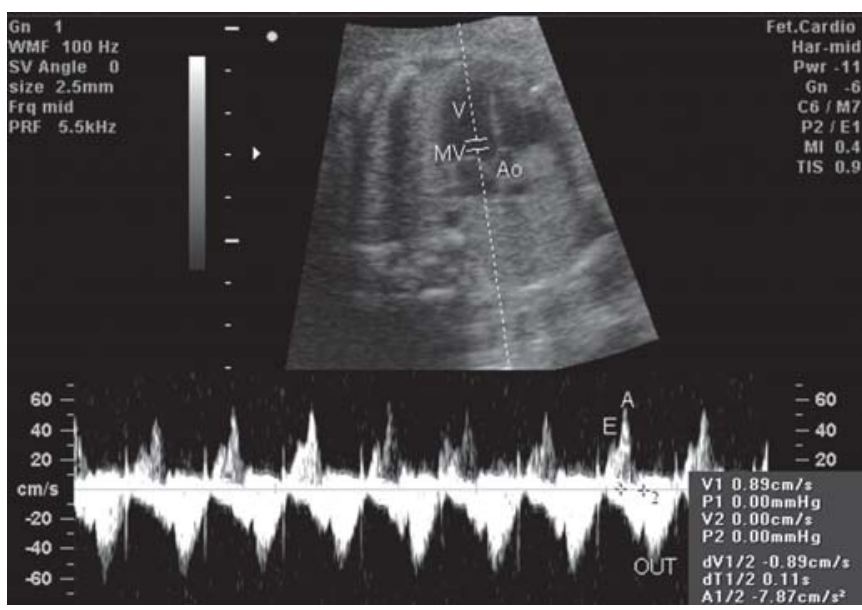

Fig. 1: MV-Ao Doppler method to measure mechanical PR interval from the intersection of the mitral $E$ and $A$ waves to the beginning of the ventricular ejection wave. E: E wave, A: A wave, OUT: aortic ejection wave, MV: mitral valve, Ao: aortic wave, V: ventricle

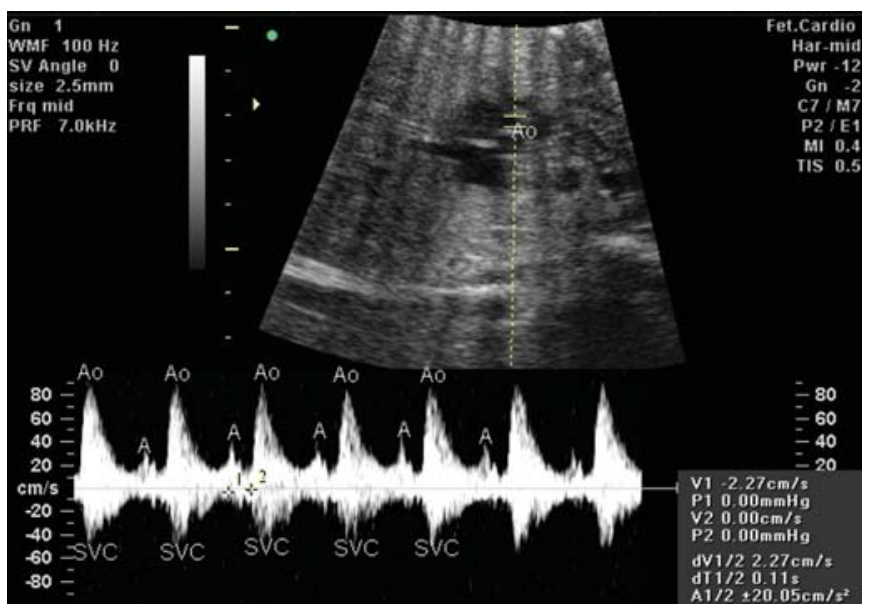

Fig. 2: AA/SVC Doppler method. A: A wave in the superior vena cava, retrograde wave from the right atrium, Ao: aortic flow, SVC: blood flow in the superior vena cava 
volume was placed in the peripheral lung vessels guided by color flow mapping on a four-chamber section of the fetal heart. Atrial and ventricular systoles were identified from the pulmonary venosus and arterial signals respectively. ${ }^{13}$

Doppler derived mechanical PR interval raise the possibility of refining the prenatal diagnosis of $\mathrm{AV}$ conduction abnormalities. A PR interval of $>150 \mathrm{~ms}$ on Doppler, FMCG or postnatal ECG has been determined to be prolonged. PR intervals can vary in the fetus as they do in neonates, or that at birth, changes in AV conduction or autonomic nervous system activity alter the PR interval. In a study, to control the usefulness of measurement of mechanical PR interval, in newborn infants Doppler tracings were obtained from the mitral valve/aortic outflow and the superior vena cava/ascending aorta, an ECG was recorded simultaneously. Close positive linear relationships to the electrocardiographic PR interval were demonstrated for the MV-Ao and SVC-Ao approaches. Both techniques overestimated the PR interval; the new MV approach using mitral closure for the same purpose did not overestimate the PR interval, but there was a trend to underestimation of the PR intervals as time intervals increased. MV recordings only need insonation of a single valve, and are thus easier to obtain. In cases with AV time prolongation the SVC-Ao method seemed superior. Higher heart rates have been demonstrated to result in increasing fusion of the E-and A-waves in the mitral valve, overlapping the beginning of the A-wave and leading to an artifactual shortening of the A-V time interval. As the PR interval gets longer with no associated decrease in heart rate, the Awave will have an earlier start in relation to the $\mathrm{E}$-wave, in turn causing increasing overlapping of the E-wave and an artifactual shortening of the A-wave. ${ }^{14}$

The newer technique of tissue velocity Doppler imaging may be helpful when evaluating the fetus with borderline findings and negative maternal anti-Ro/anti-La antibodies. ${ }^{15}$ With Tissue Doppler method the best correlation was found between postnatal PR time and prenatal PR time with measurement of right ventricular Aa-IV interval (Aa: atrial contraction, IV isovolumetric contraction). ${ }^{16}$ With a method based on raw scan-line tissue velocity data acquisition and analysis, the data are available from high-frame-rate 2D tissue velocity images and allow simultaneous sampling of right and left atrial and ventricular wall velocities to yield precise temporal analysis of atrial and ventricular events. Using this timing data, a ladder diagram like "fetal kinetogram" was developed to diagram and diagnose arrhythmias. This technique was found to be feasible and fast. ${ }^{17}$

Conventional methods of fetal cardiac function assessment are the ejection fraction (EF) for systolic function and the E/A index [(ratio of transmitral flow during early $(\mathrm{E})$ ventricular filling to flow during atrial (A) contraction)] for diastolic function, both normal population and in a population at risk for cardiac failure. The myocardial performance index (MPI, or Thei-index) and EF were found to be independent of gestational age, whereas the E/A index and isovolumetric relaxation time (IRT) as an index of diastolic function-increased with gestational age. MPI is an indicator of the systolic component of fetal left ventricular function that can be easily acquired and reproduced.

\section{IRREGULAR RHYTHM}

The most common fetal rhythm anomaly is the irregular rhythm, and usually an obstetricians or midwifes are who diagnoses it at a routine pregnancy follow-up. The causes of irregular rhythm could be most often premature atrial contractions, premature ventricular contractions or tachyarrhythmias with atrioventricular block. The irregular cardiac rhythm is not benign in the fetus. The occurence of supraventricular tachycardia in $0.5-1 \%$, the findings of structural heart disease or congenital long QT syndrome in $2.5 \%$ of the patients from studies on irregular rhythm patients make through assessment of AV conduction in an apparently healthy fetus necessary.

\section{EXTRASYSTOLES}

Extrasystoles are most often premature atrial contractions (PACs), what are usually identified in third trimester fetuses and their frequency may be highly variable. They may be conducted (Fig. 3) or blocked (Fig. 4) at the atrioventricular (AV) node. These are usually benign, resolving just before or shortly after birth. The follow-up is necessary, because some (1-3\%) of affected fetuses have intermittent runs of supraventricular tachycardia. PACs identified with cardiomegaly, evidence of ventricular systolic dysfunction, AV valve regurgitation and/or hydrops could be signs of a temporary supraventricular tachycardia (SVT). Each third day control of the fetal heart frequency, weekly or biweekly obstetrical ultrasound assessment is warranted until they have resolved or until delivery.

Less than $10 \%$ of affected fetuses have associated structural heart defect-lesions associated with significant mitral or tricuspid insufficiency atrial dilatation, aneurysm like dilation of the foramen ovale flap, intracardiac tumors (Fig. 5). In such fetuses the minor hemodynamic alterations that occur with the extrasystoles may not be tolerated.

Premature ventricular contractions are rare (Fig. 6). They are usually benign. It is necessary to rule out myocardial disease or intracardiac tumors. Prenatal and postnatal follow-up is 


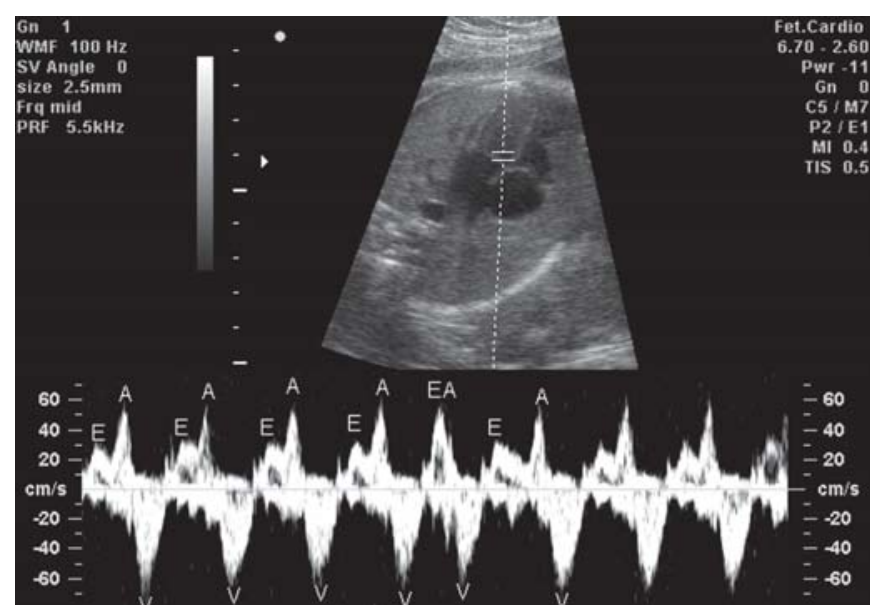

Fig. 3: Conducted premature atrial beat. E: E wave, A: A wave, EA: fused $E$ and $A$ wave because of premature beat

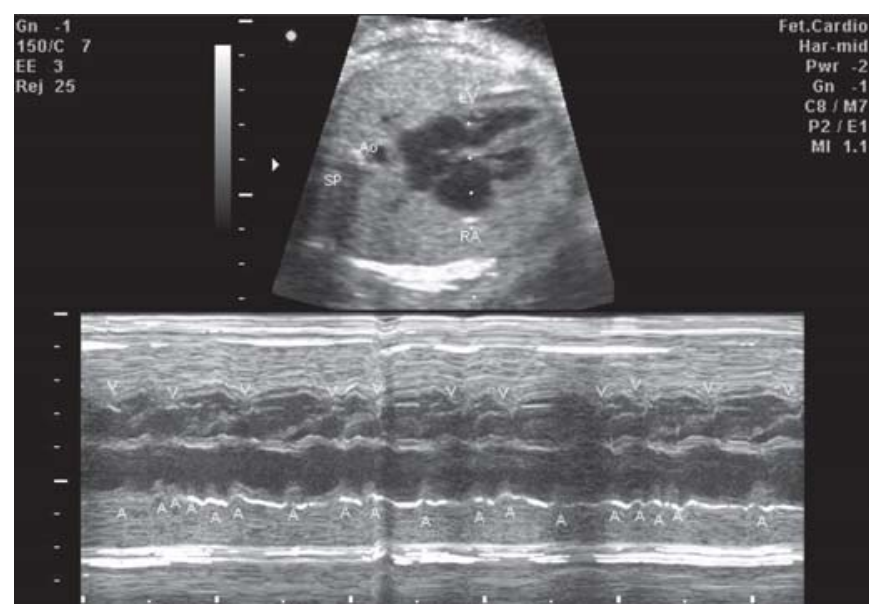

Fig. 4: Blocked premature atrial beats, M-Mode method. A: atrial contraction, V: ventricular contraction

always indicated to confirm the nature of the ectopy and exclude intermittent runs.

\section{FETAL TACHYARRHYTHMIAS}

The definition of the tachycardia is, when the fetal heart-rate exceeds $180 \mathrm{bpm}$.

\section{VENTRICULAR TACHYCARDIA}

Ventricular tachycardia is rare during fetal life. With echocardiography (M-mode, pulsatile-Doppler, tissue-Doppler) in the setting of fetal tachycardia the findings of atrioventricular dissociation with a ventricular rate that is faster than the atrial rate suggests ventricular tachycardia. ${ }^{18}$ If there is $1: 1$ retrograde conduction it is impossible to distinguish between ventricular and supraventricular tachycardia.

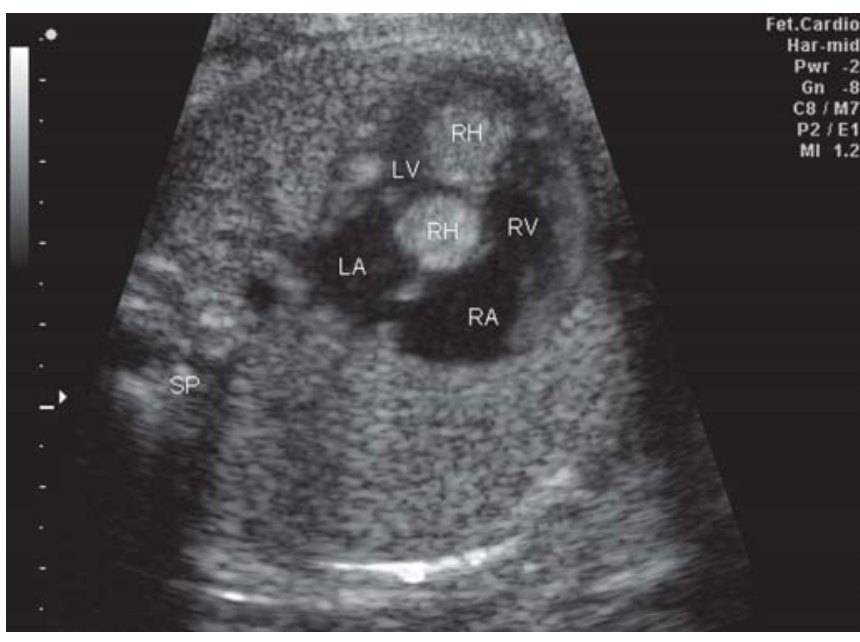

Fig. 5: Multiplex rhabdomyomas in the heart. RH: rhabdomyoma, LA: left atrium, RA: right atrium, LV: left ventricle, RV:right ventricle, SP: spine

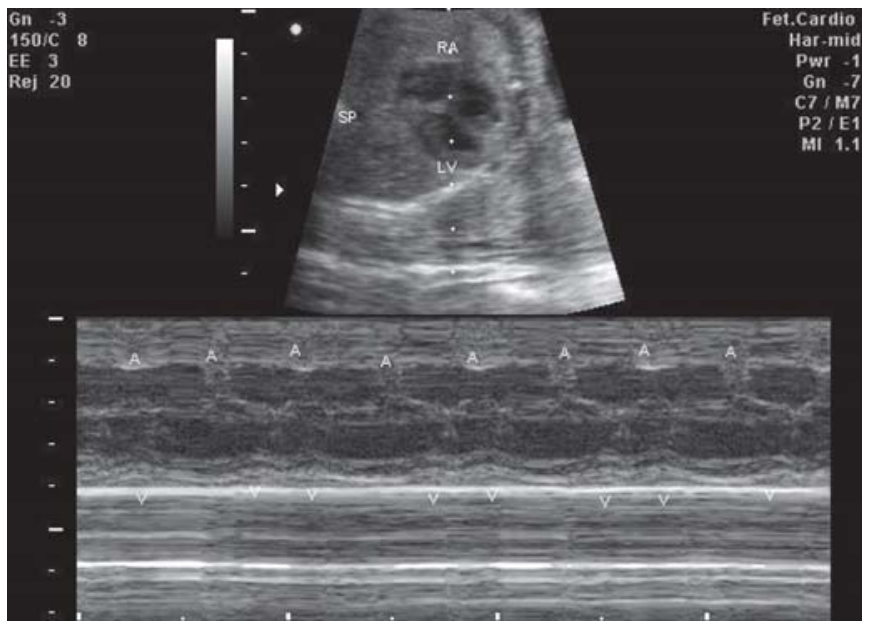

Fig. 6: Ventricular extrasystole, M-Mode method. A: atrial contraction, V: ventricular contraction

A rare presentation of fetal long QT syndrome is second degree atrioventricular block and ventricular tachycardia leading to congestive heart failure. The best method to diagnose QTC prolongation and torsade de pointes is the fetal magnetocardiography. As a sign of torsade de points, the peak-to peak intervals of ascending aorta flow during ventricular tachycardia are markedly irregular. ${ }^{14,18,19}$

The therapy of the fetal ventricular tachycardia is difficult. In cases where it is not possible to rule out the ventricular tachycardia, Digoxin therapy is contraindicated, rather amiodarone is the drug of the choice. If the diagnosis of the ventricular tachycardia is sure the pregnancy is before 34th weeks and there are signs of the decompensation, therapy with lidocain are reasonable with strong maternal control. 


\section{SUPRAVENTRICULAR TACHYCARDIA}

Supraventricular tachycardia is the most common cause of the fetal tachycardia. Fetal magnetocardiography is an excellent method to control fetal arrhythmias and could help to clarify electrophysiological patterns of initiation and termination of re-entrant fetal supraventricular tachycardia.

Five distinct patterns of initiation and 4 patterns of termination were documented, with the most common patterns of initiation involving re-entrant PACs. Wakai and coworkers were able to detect the Wolf Parkinson White syndrome, QRS aberrancy, and multiple re-entrant pathways that were crucial for defining the rhythm patterns. They observed one initiation pattern involving spontaneous antegrade block of the accessory connection in WPW and two patterns involving re-entrant PACs, one of which used a second re-entry pathway to sustain SVT. There were frequent ectopy and intermittent atrial polygeminy. They found a high incidence of accessory AV connections as opposed to AV node re-entry SVT; presence of both concealed and manifest pre-excitation; presence of prolongation of VA conduction in association with aberrant QRS conduction during SVT; an association of SVT onset with prolonged AV conduction and subsequent re-entry. FMCG actocardiography revealed an unexpectedly strong association between fetal trunk movement and the initiation and termination of SVT, suggesting that autonomic influences play a key role. ${ }^{6}$

Fouron and coworkers proposed to plan the management of the fetal tachyarrhythmia based on analysis of pulsed-Doppler recordings of fetal heart's blood flow. They determined short V-A tachycardia, when V-A (ventriculo-atrial period) was shorter than A-V (atrio-ventricular period) period (Fig. 7). Short V-A tachycardia with 1:1 AV conduction and with ventricular rates of 230-280 bpm suggests re-entrant tachycardia and they propose the digoxin as the first choice drug. Short V-A tachycardia with AV dissociation means with high probability of junctional ectopic tachycardia, which is usually resistant to digoxin and sotalol, Amiodarone could have success. AST and first and second AV block with the diagnosis of atrial ectopic tachycardia usually responds to sotalol.

Long V-A tachycardias are seen in sinus tachycardia, in permanent junctional reciprocating tachycardia or in atrial ectopic tachycardia. The first choice drug is sotalol. In intraatrial re-entrant tachycardia with or without block, the first choice drug is digoxin. ${ }^{10,20}$

In a large, retrospective review of 11 papers, with 485 cases Krapp and coworkers have find that atrial flutter accounted for 26.2\% of all cases of fetal tachyarrhythmias and supraventricular tachycardia for $73.2 \%$. Hydrops fetalis occurred in $38.6 \%$ in atrial flutter and $40.5 \%$ in SVT patients. Hydropic patients had

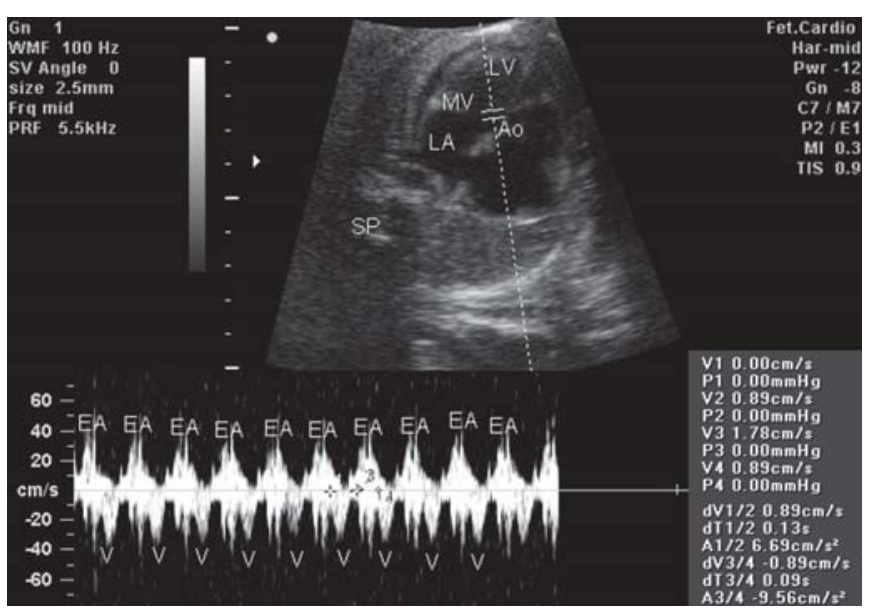

Fig. 7: Long V-A tachycardia. The V-A interval is $130 \mathrm{~ms}$, the A-V interval is $90 \mathrm{~ms}$. EA: atrial inflow, V: ventricular outflow, MV: mitral valve, Ao: aorta, LV: left ventricle, LA: left atrium, SP: spine

higher ventricular rates (median: 240 bpm, range 240-300 bpm) than nonhydropic fetuses (220 bpm, range 200-310), the atrial rates were not significantly different. Digoxin resulted in a higher conversion rate in nonhydropic fetuses. The mortality of atrial flutter was similar to that of supraventricular tachycardia at $8.0 \%$ versus $8.9 \%{ }^{21}$ The sotalol was found to be useful in transplacentar treatment of atrial flutter and supraventricular tachycardia.

In study of Oudijk and coworkers eighteen fetuses were studied, nine with atrial flutter and nine with supraventricular tachycardia, to determine the transplacentar passage of sotalol. The daily maternal sotalol dose was linearly related to the maternal plasma concentration. The mean fetal/maternal sotalol plasma concentration was 1.1 (range 0.67 to 2.87 , SD 0.63 ), and the mean amniotic fluid/fetal blood ratio of sotalol was 3.2 (range 1.28 to 5.8, SD 1.4). The effectiveness of sotalol therapy could not be extrapoled from maternal blood levels. They found to be large difference in sensitivity of sotalol. Sotalol seemed to be more efficacious than other drugs in fetal atrial flutter (AF). As proarrhythmia is known dose related, low initiation doses are preferable and dosage increases should be stepwise. Close monitoring, especially during the initiation phase, is recommended. In addition maternal potassium levels and magnesium levels should be monitored regularly and supplemented if necessary. The protocol in AF: $80 \mathrm{mg}$ of Sotalol twice daily, stepwise increased with $80 \mathrm{mg}$ per three days to a maximum of $160 \mathrm{mg}$ three times daily. The maternal toxic level is $2.5 \mathrm{mg} / \mathrm{l}$ at which marked QTc prolongation is noted. The F/M ratio of 1.1 shows that the fetus also stays below the "toxic"level. ${ }^{22}$ In cases with fetal supraventricular tachycardia and hydrops fetalis flecainide could control the fetal arrhythmia. 
The therapeutic range is 200-700 $\mu \mathrm{g} / \mathrm{l}$. Strong maternal ECG and flecainide concentrations need to be checked out to term to avoid potential maternal and fetal toxicity. There is no evidence that flecainide is concentrated in the fetus. ${ }^{23}$

In a large study on 127 consecutive fetuses in a single tertiary center from 1980-1998, the data of 105 fetuses with SVT and of 22 fetuses with AF were analyzed, 52 were hydropic, 75 nonhydropic. Prenatal control of the tachycardia was achieved in $83 \%$ of treated nonhydropic fetuses compared with $66 \%$ of the treated hydropic fetuses. First line drug treatment for hydropic fetuses were: digoxin, digoxin + verapamil, flecainide. Digoxin monotherapy was a poor choice for the hydropic fetuses. ${ }^{24}$

From the charts of 19 fetuses born at St. Justine hospital with sustained supraventricular tachycardia, three infants developed cholestasis. They survived and resolved their cholestasis. At all three patients with cholestasis fetal hydrops was presented. Two potential mechanisms can be involved: congestion or ischemia. Significant venous congestion classically seen in cardiac failure and secondary hydrops is a major element for the appearance of postnatal cholestasis. In tachycardia with a fast conducting re-entrant pathway, atrial contractions occur during ventricular ejection, thus against closed AV valves. Tall reverse " $A$ " waves are present in the venous system of fetuses with re-entrant supraventricular tachycardia. This type of arrhythmia could induce backward venous congestion and hydrops, even in absence of myocardial failure. The duration of the process also play a significant role in the development of impaired bile flow. ${ }^{25}$

\section{ATRIAL FLUTTER (AF)}

Fetal atrial flutter is usually identified late in gestation. Atrial flutter is associated with atrial rates ranging from $300-550 \mathrm{bpm}$ with variable AV conduction and ventricular rates. The atrial rate is confirmed through M-mode (Figs 8 and 9) or systemic venous Doppler demonstration of the atrial wave rates.

Lukas and coworkers studied forty-five patients with documented AF. Fetal hydrops was seen in 20 patients. 17 received maternal therapy, 2 were delivered and 1 was not treated because it had a severe nontreatable cardiac malformation. In the nonhydropic group of 24 patients 18 were treated and the remaining 6 were delivered immediately. In the hydropic group 10 received single drug (digoxin or sotalol) therapy and 7 received multidrug therapy. In the nonhydropic group 13 received a single drug (digoxin or sotalol) and 5 received multidrug therapy. One patient with rapid 1:1 atriventricular conduction (heart-rate $480 \mathrm{bpm}$ ) died in utero and another died due to a severe hydrops and stenosis of the venous duct. Of the 43 live-born infants 12 (18\%) were in AF at birth. Four

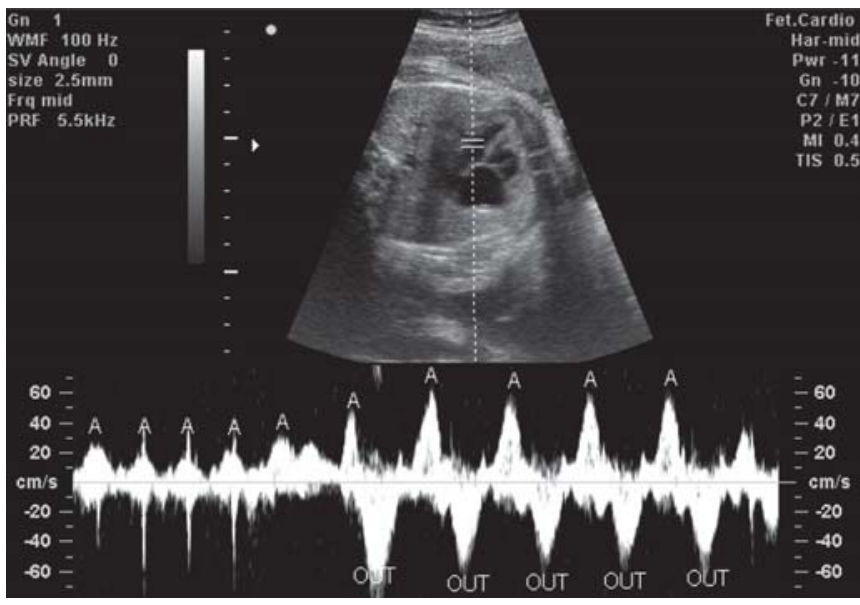

Fig. 8: Termination of atrial flutter. A: A wave, OUT: ventricular ejection wave

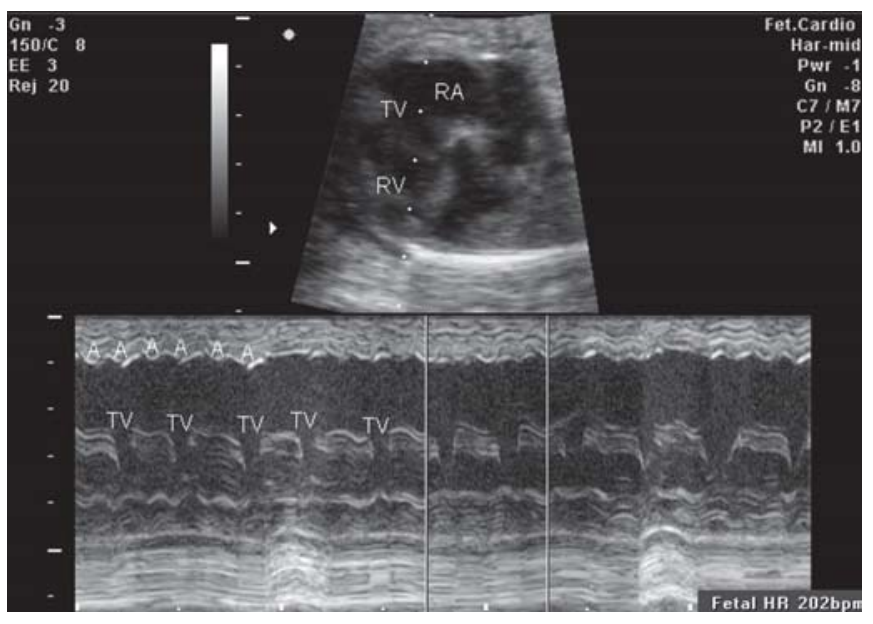

Fig. 9: Atrial flutter with 1:2 block. M- mode method. A: atrial contraction, TV: opening of the tricuspid valve, RA: right atrium, RV: right ventricle

patients with fetal flutter and hydrops showed significant neurological pathology immediately after birth. Three of the 20 hydropic patients were not treated, one due to an associated hypoplastic left heart syndrome, and the other two were delivered soon after diagnosis because they were near term. Six of nonhydropic fetuses received no medication, as they were delivered within a week of diagnosis and beyond 35 weeks of gestation. Action potential prolongation in the absence of conduction slowing seems more effective in termination human AF than depression of the excitability. Sotalol (class III drug) has excellent transplacental passage in a dose regimen of 160 mg twice daily. Monitoring of the maternal QT interval is essential in excluding maternal proarrhythmia. Amiodarone (class III drug) has been used for fetal arrhythmias but has many side effects does not cross the placenta as readily as does sotalol. The treatment with sotalol as the first drug of 
choice is more reasonable because digoxin transplacentar passage is slower, relapse into AF occurs more often at birth, and subjective maternal compliants are prominent than with sotalol. $^{26}$

The early morbidity of atrial flutter is significant, but the long-term prognosis is excellent. ${ }^{27}$ There are some case reports on probable inherited ethiology of fetal atrial flutter. ${ }^{28}$

\section{FOLLOW-UP}

Follow-up study of 292 fetuses with various cardiac arrhythmias were to estimate the long-term outcome. There were analyzed 200 cases of atrial extrasystoles, 35 cases of atrial tachycardias, 36 cases with atrioventricular block, 14 patients with sinus bradycardia, and 7 of ventricular extrasystoles. The incidence of cardiac anomalies was $12 \%$ in the study population. In utero cardiac failure was noted in $11 \%$. Among fetuses with atrial extrasystoles 1\% developed supraventricular tachycardia after birth. During antiarrhythmic therapy sinus rhythm was achieved in $92 \%$ of nonhydropic and $63 \%$ of hydropic fetuses. The latter had higher mortality and risk for neurological morbidity than did nonhydropic fetuses, $38 \%$ versus $3.7 \%$ and $40 \%$ versus $12 \%$ respectively. In the follow-up of the whole study population lasting a median 5 years, 93\% are alive and 3\% have a neurological disorder. All fetal arrhythmias except atrial extrasystoles were associated with a moderately high-risk for fetal distress. In cases of compromise, fetal and neonatal prognosis was poor and was an indication for perinatal medication. After a newborn period the prognosis has been good. However the risk of neurological morbidity must be taken into consideration. ${ }^{29}$

SVT results in reduced diastolic filling time which combined with the normal limitations of relaxation and reduced compliance of the fetal myocardium leads to increasing atrial and central venous pressures, in the evolution of placental edema and dysfunction which leads to fetal hypoxemia. Long standing and/or fluctuating cerebral perfusion as a result of intrauterine $\mathrm{AF}$ is responsible for the disturbance of the maintenance of adequate cerebral perfusion. It is exposing the brain to ischemia with increased risk of intracranial hemorrhage. It is opted to treat all fetuses with $\mathrm{AF}$ at time of presentation, unless immediate delivery is possible. The stress of the delivery and release of catecholamines may have an influence on retriggering the onset of AF, such seen in SVT.

Treatment of the SVT is proposed for fetuses with risk of signs of heart failure-incessant SVT, early onset of SVT $(<32$ weeks). Altered maternal gastric emptying and increased maternal renal clearance could necessitate the use of higher doses. In the absence of effective therapy the mortality of the hydropic fetuses with SVT approaches 50\%, with treatment the mortality is $10 \%$. It is recommend the maternal hyperglycemic state in the acute resuscitation of the hydropic fetus with supraventricular tachycardia which in providing metabolic support for the fetal myocardium, may ultimately be used coincident with initiation of antiarrhythmic treatment. It is important to control the mother, maternal heart-rate, serial ECGs and telemetry and monitoring drug concentration. ${ }^{30}$

\section{BRADYCARDIA}

\section{Sinus Bradycardia}

Persistent fetal sinus bradycardia is a rare condition and has been reported with central nervous system abnormalities, maternal treatment with beta blockers, excessive vagal tone, hydrops, neonatal long QT syndrome and intrauterine growth retardation. Sinus bradycardia has also been associated with complex heart defects in which absence or hypoplasia of the sinus node is a typical finding (Figs 10 and 11). ${ }^{31}$

Sinus bradycardia has been reported recently as a possible sign of maternal anti-SSA/Ro antibodies. In animal model knockout of the neuroendocrine $\mathrm{alfa}_{1 \mathrm{D}} \mathrm{Ca}$ channel in mice resulted in significant sinus bradycardia and atrioventricular block (CAVB), a phenotype reminiscent to that seen in CAVB. There was tested the hypothesis that the alfa $\mathrm{1D}_{1 \mathrm{D}} \mathrm{Ca}$ channel is a novel target for positive IgG. It was demonstrated that the alfa $\mathrm{ID}_{1 \mathrm{D}}$ Ca channel protein is expressed in human fetal heart, the positive IgG inhibited the alfa $\mathrm{ID}_{1 \mathrm{D}} \mathrm{I}_{\mathrm{Ca}-\mathrm{L}}$, and there was a direct crossreactivity of positive IgG with the alfa $\mathrm{a}_{1 \mathrm{D}} \mathrm{Ca}$ channel protein. Given that alfa $\mathrm{ID}_{\mathrm{D}} \mathrm{I}_{\mathrm{Ca}-\mathrm{L}}$ activates at voltages within the pacemaker's diastolic depolarization, inhibition opens new directions in the development of pharmatherapeutic approaches in the management of CAVB. ${ }^{32}$

Prenatal sinus bradycardia or recognition of 2nd degree AV block may lead to early detection and treatment of long QT syndrome. QTc prolongation was found to be more common in utero than postnatally, but it does not necessarily imply that fetal QTc prolongation is a benign condition. Several studies of newborns have suggested that QT prolongation and the concomitant increased risk of ventricular tachyarrhythmias may account for the significant mortality. QT prolongation was highest in the fetuses with the poorest outcome and was often accompanied by $\mathrm{T}$-wave alternants. Common genetic polymorphisms have been shown to alter the QT interval, and prenatal molecular genetic testing for long QT syndrome has been demonstrated. The recently reported environment-genetic interactions could result arrhythmias or sudden infant death syndrome. ${ }^{4}$ Few cases of fetal ventricular tachycardia due to 


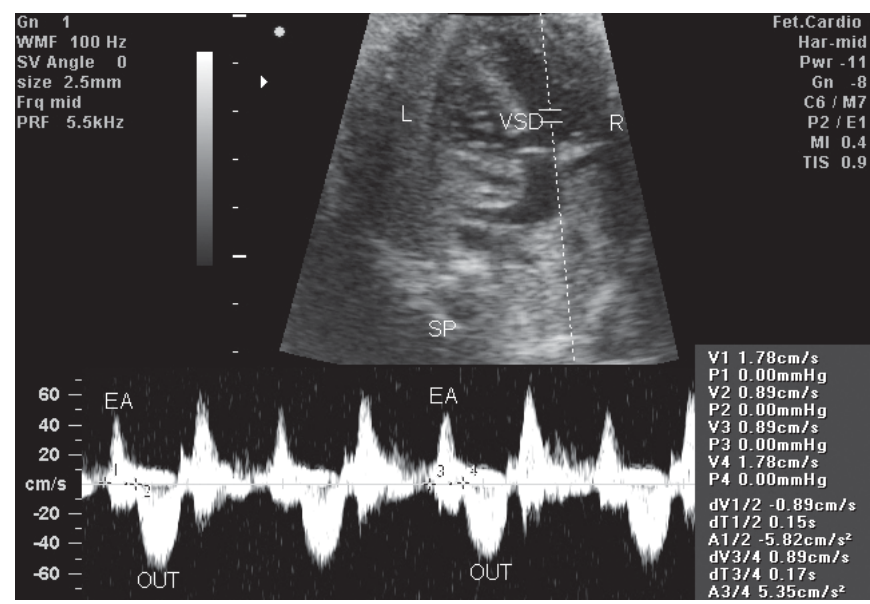

Fig. 10: $L$ transposition, ventricular septal defect and 2nd degree AV block. The PR interval is $150-170$ ms. Each second atrial beats are nonconducted. EA: fused $E$ and $A$ waves, OUT: ventricular ejection wave, L: left, R: right, VSD: ventricular septal defect, SP: spine

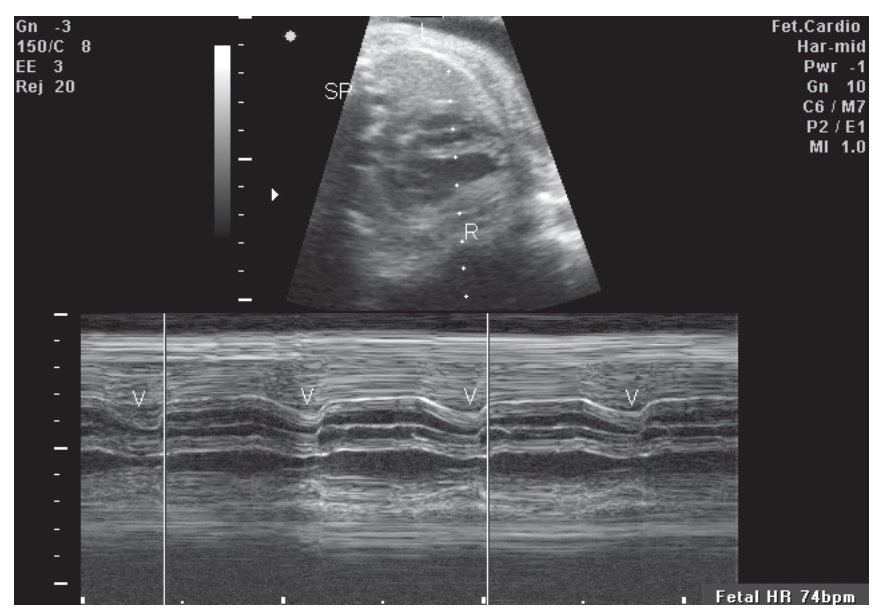

Fig. 11: $L$ transposition. M-mode. The ventricular rate is $74 \mathrm{bpm}$. SP: pine, V: ventricular contraction. L: left, R: right. The anatomical right ventricle is visible on the left side, the left ventricle on the right side

congenital long QT have been reported in the literature. Some cases presented fetal ventricular tachycardia with both bradycardia and AV block. ${ }^{14,18,19}$

\section{CONGENITAL ATRIOVENTRICULAR BLOCK (CAVB)}

The neonatal lupus is the model of passively acquired autoimmunity. The mean histologic lesion of autoimmuneassociated CAVB is fibrosis of the conducting tissue, and in some cases the surrounding myocardium. The mechanism by which maternal anti-SSA/Ro-SSB/La antibodies initiates and perpetuated inflammation and eventuate in scarring of the atrioventricular node, is not yet defined. In vitro and in vivo studies suggest that one pathologic cascade leading to scaring may be initiated via apoptosis, resulting in translocation of SSA/Ro-SSB/La antigens and subsequent surface binding by maternal autoantibodies. It was found that apoptosis was increased in available sections including septal tissue (containing the conduction system), the right ventricle and left ventricle from two fetuses dying with CHB compared to agematched normal abortuses from elective termination. Apoptotic cardiocytes were not present in contiguous tracts, but rather were diffusely scattered between nonapoptotic cells. Human IgG was colocalized to the apoptotic cells.

In CAVB the maternal anti-SSA/Ro-SSB/La antibodies result in opsonization and inflammatory/fibrotic squeal. These opsonized cardiocytes are phagocytosed by macrophages, which secrete factors those transdifferenciate fibroblasts into myofibroblasts, a scarring phenotype. ${ }^{33}$ There are theories, that the development of CAVB is a two stage process. In the first step, the maternal autoantibodies are transferred through the placenta and bind structures in the developing fetal heart, leading to first-degree AV block. This state is spontaneously reversible with further maturation of the fetus. A second phase is entered and progression to complete heart block commences. Factors involved in this second phase may be mainly fetal and related to the immunogenetic profile of the individual. A predisposition to an intense inflammatory reaction to the antibody deposition might lead to the reaction to the subsequent mononuclear cell infiltration and fibrosis, with a permanent injury as the outcome.

CAVB occurred in $2.6 \%$ of a large population of fetuses evaluated for an irregular rhythm. The high prevalence of fetal first degree AV block with normal postnatal PR intervals in a group of fetuses with maternal anti-Ro/La antibodies has been proposed due to a transient biological response to the transplacentar transfer of Ro/La antibodies. ${ }^{3}$

If 2nd degree AV block is present, transplacentar steroid treatment theoretically may limit progression. Prophylactic steroid therapy in the fetus without documented AV conduction disease is not warranted due to maternal and fetal side effects. After a high-dose steroid therapy for fetal heart block several maternal complications were found including diabetes, hypertension, infections, bone density loss and cataract. Fetal risks were oligohydramnios and growth restriction, including the head and possibly central nervous system injury without clear evidence of benefit to fetal cardiac conduction. ${ }^{7}$

Early detection of incomplete AV block successfully identifies a group at highest risk developing permanent AV block. Treating this group may incur greater benefit than risk. The anti-inflammatory effects of dexamethasone might have interrupted on-going damage to the conduction system 
secondary to maternal autoantibodies. The Doppler mechanical PR interval does not distinguish between electrical PR interval prolongation due to $\mathrm{AV}$ conduction delay and abnormalities of interatrial conduction. These limitations in Doppler mechanical PR interval measurements can be overcome if FMCG is available. The newer technique of tissue velocity Doppler imaging may be helpful when evaluating the fetus with borderline findings and negative maternal anti-Ro/anti-La antibodies.

The echocardiographic findings of a mechanical PR interval of $>150 \mathrm{~ms}$ at any gestational age should prompt a search for maternal anti-Ro/anti-La antibodies and close follow-up for other complex arrhythmias (Figs 12 and 13). ${ }^{11,12,15}$ There were prospective studies on group of fetuses with maternal anti-Ro/ La antibodies, to diagnose early signs of CAVB with measuring fetal PR interval. The results are contradictory and need further studies on this.

In one of these studies on twenty-four women with antiSSA/Ro 52-kd antibodies and consequently increased risk for fetal heart block were followed up weekly, between 18 and 24 weeks of gestation, with echocardiography. The fetal A-V time intervals were longer and heart-rates were slightly lower compared with those in controls. Eight of 24 fetuses had signs of first-degree block. The definition of a prolonged PR interval was set at $135 \mathrm{~ms}$ (2SD above their mean, derived from their previous studies). One of these fetuses had progression to complete block, and another showed recovery from seconddegree block to first-degree block with dexamethasone treatment. In the remaining six fetuses, spontaneous normalization occurred before or shortly after birth. Fetuses with normal AV time intervals at 18-24 weeks had normal electrocardiographic results at birth. Anti-Ro antibodies have effects on calcium currents in cardiocytes. In 2 of 7 babies who died of CAVB, the sinuatrial nodes were hypoplastic and, in some of these cases surrounded by extensive fibrosis. Sinus bradycardia has been described in infants of mothers with anti-Ro antibodies. ${ }^{33}$

In another prospective study, one hundred twenty-seven pregnant women with anti-SSA/Ro antibodies were enrolled. The protocol included fetal echocardiograms performed weekly from 16 to 26 weeks' gestation and biweekly from 26 to 34 weeks. RP intervals $>150$ ms were considered prolonged, consistent with first-degree block. Ninety-two fetuses had normal PR intervals. Neonatal lupus developed in 10 cases, 4 were neonatal lupus rash only. Three fetuses had third-degree block; none had a preceding abnormal PR interval, although in 2 fetuses $>1$ week elapsed between echocardiographic evaluations. Tricuspid regurgitation preceded third-degree block in 1 fetus, and an atrial echodensitiy preceded block in a second. From the three cases of third degree block, none were reversed by

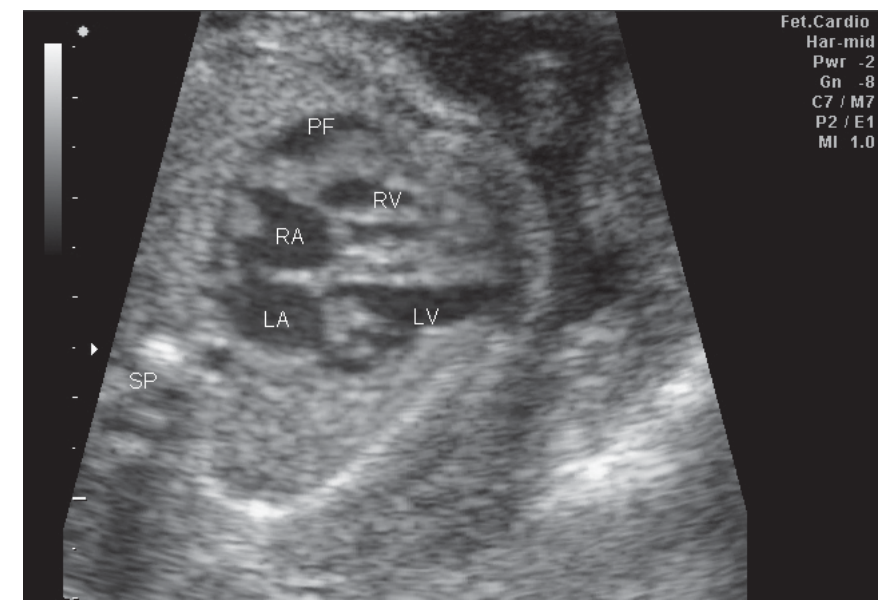

Fig. 12: Cardiomegaly at a fetus with CAVB because of maternal autoantibodies. Mild pericardial fluid (PF), mild myocardial fibrosis. SP: spine, RA: right atrium, LA: left atrium, RV: right ventricle, LV: left ventricle

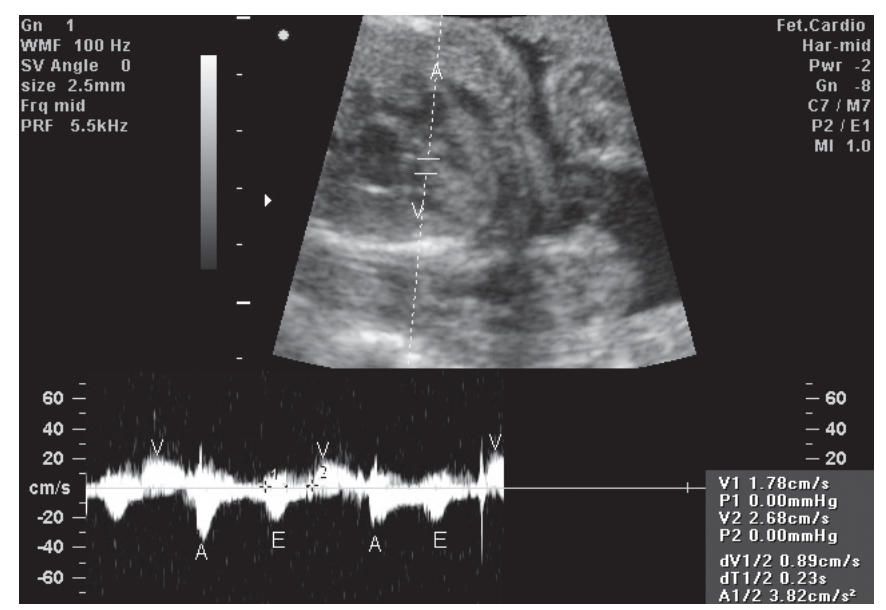

Fig. 13: Complete atrioventricular dissociation. The PR interval is 230 ms. EA: fused $E$ and $A$ waves, $V$ : ventricular ejection wave, $A$ : right atrium, $V$ : left ventricle

dexamethasone. Two fetuses had PR intervals $>150 \mathrm{~ms}$, detected at or before 22 weeks, and each reversed within 1 week with 4 mg dexamethasone. The ECG of 1 additional newborn revealed a prolonged PR interval persistent at 3 years despite normal intervals throughout gestation. No first-degree block developed after a normal ECG at birth. Heart block occurred in 3 of 16 pregnancies (19\%) in mothers with a previous child with congenital heart block and in 3 of 74 pregnancies (4\%) in mothers without previous child with congenital heart block or rash.

Advanced block and cardiomyopathy can occur within 1 week of normal echocardiogram without initial first-degree block. This more extensive injury can occur in utero or postnatally, even as late as 9 years of age. May be that PR prolongation 
represents a variant of a normal, and only in retrospect does it have clinical significance if it either is sustained after birth or progresses to more advanced block. The prolongation of the fetal Doppler mechanical PR interval was not a definitive tool to detect early signs of anti-AAS/Ro associated CAVB. ${ }^{34}$

\section{TREATMENT POSSIBILITIES IN CAVB}

Although the prenatal use of corticosteroids, plasmapheresis, sympathomimetics and either prenatal pacing or early delivery with postnatal pacing has been proposed, the true efficacy of therapy is not clear.

Current approach to affected pregnancies is to initiate dexamethasone therapy at diagnosis of fetal AV block (4 mg/ die) and to ad maternal sympathomimetics for ventricular rates of $<55$ beats/min. Weekly-biweekly fetal echocardiograms are used to follow the progress, and an elective delivery by cesarean section at 36 to 37 weeks is planned. If there are signs of fetal compromise, it is proposed an earlier delivery with aggressive postnatal management, including eraly pacemaker placement. When there is oligohydramnios, maternal dexamethasone is tapered off; however, persistence of reduced amniotic fluid may prompt earlier delivery. ${ }^{35}$

Prednisone and prednisolone are inactivated by placenta hydroxilases and only less than $10 \%$ of the mother blood level can reach fetus, dexamethasone and betamethasone cannot be inactivated. Some data suggest that prolonged fetal exposure to dexamethasone may impair cerebral development, but there are reports on children treated with dexamethasone prenatally (cumulative dosage 20-260 mg), who were tested postnatally with different scales according to their age and they were found with normal intelligence quotient. ${ }^{36}$

Intravenous immunoglobulin (IVIG) is efficient in various immune mediated conditions. It seems conceivable to employ IVIG in cases of CAVB or neonatal lupus, either administered to the mother or directly through cordocentesis in the treatment of the fetus and neonate with reduced myocardial function suggesting more diffuse disease. ${ }^{37}$

\section{CAVB - FOLLOW-UP AND ASSOCIATED DISEASES}

In a large study on 87 infants whose mothers had anti-Ro antibodies were followed prospectively, 47 had neonatal lupus syndrome. Five of the 47 infants with neonatal lupus syndrome and 2 of the healthy infants had hydrocephalus, resulting in a prevalence of $8 \%$ in the entire cohort. This is significantly higher than the prevalence in the general population of $0.048-0.081 \%$. It seems to be possible, that hydrocephalus and macrocephaly are manifestations of neonatal lupus syndrome and infants born to mothers with anti-Ro antibodies should be carefully monitored for hydrocephalus as part of their routine physical examination. ${ }^{38}$

In a Justine hospitals between 1993 and 2001 - 5 newborns were born with CAVB, three of these presented with cholestasis, 2 of these infants died. Clinically severe and irreversible liver failure could be observed. Histologically extensive collapse of the stroma and the absence of hepatocytes (foie vide) were observed. At all three patients' fetal hydrops was presented. Cholestasis has been described in patients with neonatal lupus erythematosus without AV block. Liver inflammation could cause cholestasis. ${ }^{25}$

When complete congenital atrioventricular block (CAVB) is associated with endocardial fibroelastosis (EFE), there is a very high mortality rate. The ethiology of EFE remains unclear, but it may be a result of an autoimmune process. Some patients with anti-Ro and anti-La antibody-associated CAVB have developed EFE despite adequate pacing at psysiologic heart rates, implying that perhaps EFE and CAVB are two separate disease manifestations of neonatal lupus erythematosus.

Nield et al describe one fetal and two infant cases with EFE and cardiomyopathy with clinically normal conduction systems conceived by mothers positive for anti-Ro and/or anti-La antibodies. At histological examination severe EFE predominantly in the left ventricle, where it was diffuse and thick (1 mm depth), and in the right ventricle focal mild EFE was found. At another patient echogenicity involved both atria and the sinus node and atrioventricular node were calcified. Immunohistochemical findings were as: LV tissue was strongly and diffusely positive for immunoglobulin IgG, moderately positive for IgM and mildly positive for IgA deposition. Neonatal lupus erythematodes (NLE) involves heart, skin and liver in patients born to mother of anti-Ro, anti-La. Antibodies deposit not throughout the myocardium, and not just within the conduction system. There is IgM and occasionally IgA deposition, a T-cell and to a lesser degree, a B-cell infiltrate. There is an active fetal B-cell response in addition to the passive deposition of transplacentally passed maternal immunoglobulin. The initial myocardial damage in utero is likely secondary to the passive deposition of maternal anti-Ro and anti-La antibodies, the progression of EFE may be the result of an ongoing fetal and postnatal autoimmune reaction.

The EFE accounted for over $80 \%$ of the deaths in these patients. Some cases may go undiagnosed where there is a fetal demise. Echocardiographic assessment is very subjective and often identifies only the most severe degree of EFE. Idiopathic dilated cardiomyopathy represents unrecognized maternal autoantibody-induced EFE. CAVB occurs only in 1 to $7 \%$ of pregnancies with anti-Ro and anti-La antibodies, suggesting 
that there must be additional maternal and/or fetal factors that influence disease development and manifestation. The maternal factors may be related to the antibody profile. The fetal factor could be viral infection or genetic predisposition. Prenatal maternal and postnatal infant corticosteroid therapy has been shown to improve ventricular function in some cases. The current management is limited to supportive care and antifailure medications. $^{39}$

Moak et al. studied the clinical course of 16 infants with CAVB who developed late-onset dilated congestive cardiomyopathy despite early and adequate institution of cardiac pacing. In 12 infants the CAVB was diagnosed prenatally, left ventricular function was normal in all. Four patients received medical therapy in utero: dexamethasone, terbutaline and bethamethasone. In none did the degree of AV block improve. Pericardial effusion improved or resolved in two of two patients. Elevated hepatic transaminase and thrombocytopenia were found. At seven patient mild heart defects were diagnosed. Thirteen infants developed symptoms of congestive heart failure between two weeks and 30 months associated with depressed left ventricular function. At the time of clinical presentation, nine infants had symptoms or signs of an upper respiratory tract infection, two with otitis media. One infant had a respiratory syncytial virus infection. All patients were treated with positive inotropic agents and diuretics. Clinical deterioration developed later at three patients, two children presented with symptoms or signs of an upper respiratory tract infection and gastrointestinal infection. Myocardial biopsy or histological examination of the explained heart revealed the following: myocyte hypertrophy in 11 of 16 patients, interstitial fibrosis in 11 of 16 patients and myocyte degradation in two subjects. No an active inflammatory infiltrate was detected. Four infants died from congestive heart failure. Seven patients required cardiac transplantation. An upper respiratory/ gastrointestinal infection might have reactivated a previously dormant inflammatory process. The increased rate that followed pacemaker implantation may have provoked a form of tachycardia-induced cardiomyopathy. ${ }^{40}$

There are many follow-up studies on CAVB. One of a large studie on atrioventricular block and sinus bradycardia had found, that among fetuses with atrioventricular block only, the survival rate was $82 \%$, with a heart defect, prognosis was poor, $50 \%$ of the patients survived. Sinus bradycardia and ventricular extrasystoles were associated with survival rates of $75 \%$ and $67 \%$. All fetal arrhythmias were associated with a moderately high-risk for fetal distress. In cases of compromise, fetal and neonatal prognosis was poor and was an indication for perinatal medication. The risk of neurological morbidity must be taken into consideration. ${ }^{30}$

In another study 105 mothers whose sera contain anti-SSA/ Ro or anti-SSB/La antibodies, or both, and their 113 infants diagnosed with congenital heart block (CAVB) between 1970 and 1997 were studied. Of 87 pregnancies in which sufficient medical records were available, bradyarrhythmia confirmed to be CAVB was initially detected before 30 weeks of gestation in 71. There were no cases of major congenital cardiac anatomic defects; in 14 there were minor abnormalities. Twenty-two of the 113 children died, 16 within 3 months after birth. Cumulative probability of 3-year survival was 79\%. Sixty-seven of 107 liveborn children required pacemakers: 36 within 9 days of life, 15 within 1 year, and 16 after 1 year. Forty-five of the mothers had subsequent pregnancies: 8 had another infant with CAVB (16\%) and $3(6 \%)$ had a child with an isolated rash consistent with NLS. ${ }^{41}$

In a study of Edgar et al. on outcome of isolated congenital atrioventricular block, 102 cases identified, from whom 29 were diagnosed in utero (26+-5.6 weeks), 33 as neonates (before the 28th days), and 40 as children (5.7+-4.8 years of age). Anti-Ro and/or anti-La were present in 95\% of fetal, $90 \%$ of neonatal, but only $5 \%$ of children's mothers tested. Patients with CAVB having fetal, neonatal and children diagnosis had a mortality of $43 \%, 6 \%$ and $0 \%$ respectively, in the first two decades of life. Increased mortality was associated with a fetal diagnosis of CAVB, fetal hydrops (100\%), endocardial fibroelastosis (100\%) and delivery at or before 32 weeks of gestation. Timing of pacemaker implantation differed significantly among fetuses versus neonates and neonates versus children cases. At 20 years of age only $11 \%$ and $12 \%$ of CAVB patients with neonatal or children diagnosis, respectively were not paced. CAVB detected in utero represents a more severe spectrum of the disease than encountered postnatally. ${ }^{35}$

The mortality decreased from 45 to15\% with close surveillance, use of maternally administered corticosteroids, delivery at 35-37 weeks or earlier with any evidence of fetal distress or cardiovascular compromise, neonatal cardiac intensive care management and early permanent pacemaker therapy.

\section{REFERENCES}

1. Kirby ML. Molecular embryogenesis of the heart. Pediatric and Developmental Pathology 2002;5:516-43.

2. Gittenberger-De Groot A, Bartelings MM, Deruiter MC, Poelmann RE. Basics of cardiac development for the understanding of comgenital heart malformations. Pediatric Research 2005;57:169-76. 
3. Hornberger L, Sahn DJ. Rhythm abnormalities of the fetus. Heart 2007;93:1294-1300.

4. Zhao H, Strasburger JF, Cuneo BF, Wakai RT. Fetal cardiac repolarisation abnormalities. Am J Cardiol 2006;98:491-96.

5. Horigome Hitoshi, Ogata Kuniomi, Kandori Akihiko, Miyashita Tsuyoshi, Takahashi-Igari Miho, et al. Standardisation of the PQRST waveform and analysis of arrhythmias in the fetus using vector magnetocardiography. Pediatric Research 2006;59:12125.

6. Wakai RT, Strasburger JF, Li Z, Deal B, Gotteiner NL. Magnetocardiographic rhythm patterns at initiation and termination of fetal supraventricular tachycardia. Circulation 2003;107:307-12.

7. Gardiner HM, Belmar C, Pasquini L, Seale A, Thomas M, et al. Fetal ECG: A novel predictor of atrioventricular block an antiRo positive pregnancies. Heart 2007;93:1454-60.

8. Taylor MJ, Smith MJ, Thomas M. Noninvasive fetal electrocardiography in sinleton and multiple pregnancies BJOG 2003;110:668-78.

9. Fouron JC, Proulx F, Miro J, Gosselin J. Comparison of Doppler and M-mode ultrasonography to time fetal atrial and ventricular contractions. Obstet Gynecol 2000;96:732-36.

10. Fouron JC, Fournier A, Proux F, Lamarche J, Bigras JL, et al. Manegement of fetal tachyarrhythmia based on superior vena cava/aorta Doppler flow recordings. Heart 2003;89:1211-06.

11. Glickstein JS, Buyon J, Friedman D. Pulsed Doppler assessment of the fetal PR interval. Am J of Cardiol 2000;86:236-39.

12. Adelfinger G, Fouron JC, Sonesson SE, Proulx F. Reference values for time intervals between atrial and ventricular contractions of the fetal heart measured by two Doppler techniques. Am J of Cardiol 2001;88:1433-1636.

13. Carvalho JS, Prefumo F, Ciardelli V, Sairam S, Bhide A, et al. Evaluation of fetal arrhythmias from simultaneous pulsed wave Doppler in pulmonary artery and vein. Heart 2007;93:1448-53.

14. Bergman G, Jacobsson LA, Wahren-Herlenius M, Sonesson SE. Doppler echocardiographic and Electrocardiographic atrioventricular time intervals in newborn infants: Evaluation of techniques for surveillance of fetuses at risk for congenital heart block. Ultrasound Obstet Gynecol 2006;28:57-62.

15. Cuneo BF, Strasburger JF, Wakai RT. conduction system disease in fetuses evaluated for irregular cardiac rhythm. Fetal Diagn Ther 2006;21:307-13.

16. Nil M, Hamilton RM, Fenwik L, Kingdom JCP, Roman KS, et al. Assessment of fetal atrioventricular time intervals by tissue Doppler and pulse Doppler echocardiography: Normal values and correlation with fetal electrocardiography. Heart 2006;92:1830-37.

17. Rein AJ T, O’Donnell C, Geva T, Nir A, Perles Z, et al. Use of tissue velocity imaging in the diagnosis of fetal cardiac arrhythmias Circulation 2002;106:1827-33.

18. Cuneo BF, Ovadia M, Strasburger JF, Zhao H, Petropulos T, et al. Prenatal diagnosis and in utero treatment of torsades de pointes associated with congenital long QT syndrome. Am J of Cardiol 2003;91:1395-98.
19. Takahash K, Shiraish H, Ohkuchi A, Matsubara S, Kuwata T, et al. Irregular peak-to-peak intervals between ascending aortic flows during fetal ventricular tachycardia in long QT syndrome. Ultrasound Obstet Gynecol 2009;33:990-92.

20. Joglar J, Page RL. Antiarrhythmic drugs in pregnancy Curr Opin Cardiol 2001;16:40-45.

21. Krapp M, Simpson JM, Sharland GK, Katalinic A, Gembruch U. Review of diagnosis, treatment, and outcome of fetal atrial flutter compared with supraventricular tachycardia. Heart 2003;89:913-17.

22. Oudjik MA, Ruskamp JM, Ververs T, Ambachtscher B, Stoutenbeck P, et al. Treatment of fetal tachycardia with sotalol: transplacental pharmacokinetics and pharmacodynamics. J Am Coll Cardiol 2003;4:765-70.

23. Rasheed A, Simpson J, Rosenthal E. Neonatal ECG changes caused by supratherapeutic flecainide following treatment for fetal supraventricular tachycardia. Heart 2003;89:470.

24. Simpson JM, Sharland GK. Fetal tachycardias: Management and outcome of 127 consecutive cases Heart 1198;79:576-81.

25. Sant'Anna AM, Fouron JC, Alvarez F. Neonatal cholestasis associated with fetal arrhythmia. J Pediatr 2005;146:277-80.

26. Lisowski LA, Verheijen PM, Benatar AA, Soyeur DJG, Stoutenbeek $\mathrm{P}$, et al. Atrial flutter in the perinatal age group: Diagnosis, management and outcome. J Am Coll Cardiol 2000;35:771-77.

27. Casey FA, McCrindle BW, Hamilton RM, Grow RM. Neonatal atrial flutter: Significant early morbidity and excellent long-term prognosis. Am Heart Journal 1997;133:302-06.

28. Tikanoja T, Kirkinen P, Nikolajev K, Eresmaa L, Haring P. Familial atrial fibrillation with fetal onset. Heart 1998;79:19597.

29. Boldt T, Eronen M, Anderson S. Long-term outcome in fetuses with cardiac arrhythmias. Obstet Gynecol 2003;102:1372-79.

30. Hornberger LK. Echocardiographic assessment of fetal arrhythmias Heart 2007;93:1331-33.

31. Qu Y, Baroundi G, Yue Y, Boutjidr M. Novel molecular mechanism involving alfa ${ }_{1 D}$ L-type calcium channel in autoimmune-associated sinus bradycardia Circulation 2005;111:3034-41.

32. Buyon JP, Clancy RM. From antibody insult to fibrosis in neonatal lupus - the heart of the matter Arthritis Res Ther 2003;5:266-70.

33. Sonesson SE, Salamonsson S, Jacobsson LA, Bremme K, WahrenHerlenius M. Signs of first-degree heart block occur in one-third of fetuses of pregnant women with anti-SSA/Ro 52-kd antibodies. Arhritis and Rheumatism 2004;50:1253-61.

34. Friedman DM, Kim MY, Copel JA, Davis C, PhoonCKL, et al. Utility of cardiac monitoring in fetuses at risk for congenital heart block. Circulation 2008;117:485-93.

35. Jaeggi ET, Hamilton RM, Silvermann ED, Zamora SA, Hornberger LK. Outcome of children with fetal, neonatal or childhood diagnosis of isolated congenital atrioventricular block. J Am Coll Cardiol 2002;39:130-37.

36. Tincani A, Rebaioli CB, Frassi M, Taglietti M, Gorla R, et al. Pregnancy and autoimmunity: Maternal treatment and maternal 
disease influence on pregnancy outcome. Autoimmunity Reviews 2005;4:423-28.

37. Nussinovich Udi, Shoenfeld Yehuda: Intravenous immunoglobulin-indications and mechanisms in cardiovascular diseases Autoimmunity Reviews 2008;7:445-52.

38. Boros CA, Spence D, Blaser S, Silverman ED. Hydrocephalus and macrocephaly: New manifestations of neonatal lupus erythematosus. Arthritis and Rheumatism 2007;57:261-66.

39. Nield LE, Silverman ED, Smallhorn JF, Taylor GP, Brendan J, et al. Endocardial fibroelastosis associated with maternal anti-
Ro and anti-La antibodies int he absence of atrioventricular block. J Am Coll Cardiol 2002;40:796-802.

40. Moak JP, Barron KS, Hougen TJ, Wiles HBS, Balaji S, et al. Congenital heart block: Development of late-onset cardiomyopathy, a previously inderappreciated sequela. J Am Coll Cardiol 2001;37:238-42.

41. Buyon JP, Hierbert R, Copel JA, Craft J, Friedman D, et al. Autoimmune associated congenital heart block: Demographics, mortality, morbidity and recurrence rates obtained from a national neonatal lupus registry. J Am Coll Cardiol 1998;31:1658-66. 